\title{
Effet de l'expérience sociale au cours du développement sur le comportement sexuel et la production spermatique de jeunes boucs
}

\author{
P. ORGEUR, P. MIMOUNI, B. LEBEUEF *, J.P. SIGNORET
}

INRA, Station de Physiologie de la Reproduction, Laboratoire de Comportement animal, Centre de Recherches de Tours, Nouzilly, F 37380 Monnaie

* INRA, Station Expérimentale d'Insémination artificielle, F 86480 Rouillé

\section{Résumé}

Dans l'espèce caprine, les jeunes mâles destinés à produire de la semence pour l'insémination artificielle sont classiquement élevés en cases individuelles. C'est dans ces conditions que leur comportement en collecte au vagin artificiel et leur production spermatique est la plus satisfaisante. Cependant, ce mode d'élevage implique un coût d'entretien élevé au plan de la maind'œuvre et des bâtiments.

Nous avons entrepris une série d'études (trois), portant sur 74 animaux, dont l'objectif était de connaître, chez le jeune bouc, les effets de l'élevage en groupes unisexués et hétérosexués constitués à différents âges dans la période juvénile, sur le comportement en collecte et la production spermatique, en comparaison avec des animaux élevés individuellement.

Les résultats ont confirmé que l'élevage en cases individuelles permet la meilleure expression des capacités sexuelles des jeunes boucs. La semence d'animaux élevés dans ces conditions est en effet plus facile à obtenir avec un vagin artificiel que s'ils sont entretenus en groupe. La production spermatique, notamment la qualité de semence, serait également meilleure chez les mâles en cases individuelles.

Par ailleurs, nos résultats montrent qu'une constitution précoce du groupe social (dès la naissance) a un effet favorable, à la fois sur le comportement en collecte de semence et sur la production spermatique, par rapport à une réunion postérieure à la puberté. En effet, dans ces conditions, $97 \%$ des sollicitations à la collecte sont couronnées de succès, avec un nombre total de spermatozoïdes supérieur, contre $71 \%$ si le groupe a été formé après la puberté.

Par contre, une réunion des sujets entre un et deux mois ne paraît pas préférable à une réunion plus tardive ( 2 à 4,5 mois).

La présence de femelles dans les groupes paraît préférable à leur absence.

En résumé, nos résultats indiquent que l'élevage en groupes hétérosexués est possible dans l'espèce caprine, sous certaines conditions (âge de réunion précoce, notamment), permettant d'envisager une réduction substantielle du coût d'entretien des mâles.

Les conditions de manipulation, l'habituation à l'homme, semblent importantes et devront faire l'objet d'études plus précises.

Mots clés: Caprins mâles, développement, expérience sociale, comportement sexuel mâle, production spermatique. 


\section{Introduction}

Chez de nombreux mammifères, l'organisation des conduites sociales et reproductrices de l'adulte dépend, en partie, de l'expérience acquise au cours du développement. Ce phénomène est particulièrement important chez le mâle, dans le cas du comportement sexuel.

Les perturbations sont cependant variables selon les espèces, la période, la durée, le degré et le type de privation sociale subie pendant la période juvénile.

Parmi les espèces domestiques, l'élevage moderne entraîne des contraintes importantes impliquant précisément des privations sociales telles que le sevrage plus ou moins précoce, l'isolement ou la ségrégation sexuelle.

Les perturbations qui peuvent en résulter affectent les jeunes reproducteurs. Même mineures en apparence, elles peuvent avoir des conséquences économiques graves. Ainsi chez le porc élevé en isolement, le niveau d'activité sexuelle est inférieur à celui de verrats élevés en groupe (Hemsworth et al., 1981, 1983). La ségrégation sexuelle dans la période prépubère peut, notamment dans l'espèce ovine, retarder le démarrage de l'activité copulatoire (Pretorius, 1967, Le Roux \& Barnard, 1974, Orgeur \& Signoret, 1984, Orgeur et al., 1984 a). Une orientation homosexuelle préférentielle peut en être la cause, comme Zenchak et al. (1981) l'ont montré chez le bélier, et Price \& Smith (1984) chez le bouc.

Au contraire, si la période de privation sociale ou sa sévérité sont limitées, les effets négatifs de la modification des contacts avec des congénères n'apparaissent pas, chez le jeune bélier (Orgeur \& Signoret, 1984), ou le jeune taureau (LANE et al., 1983).

Dans un autre domaine, les relations sociales qui s'établissent entre des individus élevés en groupe peuvent également influencer les performances reproductrices des animaux. Ainsi, dans l'espèce ovine, la subordination sociale est parfois un facteur d'inhibition sexuelle (LINDSAY et al., 1976 ; JeNnINGS, 1977).

Chez les Caprins, peu étudiés de ce point de vue, les jeunes boucs destinés à produire de la semence dans le cadre des opérations de mise à l'épreuve sur la descendance, sont entretenus en cases individuelles. L'élevage en groupes unisexués aboutit, lors des premiers essais de collecte, à des difficultés comportementales (DE Montigny \& LequenNe, 1975) que ne semblent pas compenser complètement, comme chez les Ovins, la présence de femelles (Orgeur et al., 1984 b).

L'objectif de nos études était de connaître les effets de différentes conditions d'élevage au cours de la période juvénile sur le comportement en situation de collecte de semence au vagin artificiel et sur la production spermatique. Ainsi des jeunes boucs en cases individuelles ont-ils été comparés à des animaux en groupes unisexués ou hétérosexués constitués à différents âges. 


\section{Matériel et méthodes}

\section{A. Animaux}

\section{Expérience 1.}

En 1985, à la Station de Physiologie de la Reproduction de Nouzilly, 18 jeunes boucs de race Alpine ont été répartis en deux groupes, dans le but de connaître l'effet de l'âge à la réunion sur leur comportement en collecte de semence et sur leur production spermatique.

- N1 = 8 sujets nés en janvier et février ont été élevés ensemble depuis le sevrage, qui est intervenu dès leur naissance ;

- N2 = 10 sujets nés en décembre-janvier et février, provenant de différents élevages, ont été réunis alors qu'ils étaient âgés de 8 à 11 mois (après puberté).

Une femelle adulte ovariectomisée a été introduite à ce moment dans chacun des groupes.

Les deux groupes ont été entretenus dans un même bâtiment et les animaux ont reçu une alimentation identique composée de céréales, de luzerne déshydratée et de foin.

\section{Expérience 2.}

En 1986, à la Station Expérimentale d'Insémination Artificielle (S.E.I.A.) de Rouillé, 27 jeunes boucs de races Alpine (17) et Saanen (10), nés entre la mi-octobre et la fin janvier ont été utilisés. Cette expérience était destinée à apprécier l'effet de la ségrégation sexuelle sur les critères comportementaux et spermatiques.

Les animaux, sélectionnés sur leur ascendance, ont été répartis en trois groupes, alors qu'ils avaient de 2 à 4,5 mois.

$-\mathrm{R} 1=9$ sujets en cases individuelles ;

$-\mathrm{R} 2=9$ sujets en 2 sous-groupes de 4 et 5 ;

- R3 $=9$ sujets en 2 sous-groupes de 4 et 5 , avec dans chacun d'entre eux une femelle du même âge.

\section{Expérience 3.}

En 1987, à la S.E.I.A. de Rouillé, 29 jeunes boucs de races Alpine (17) et Saanen (12), nés entre début novembre et début février et sélectionnés sur leur ascendance ont été répartis, en mars, en deux groupes :

- R4 = 14 sujets (les plus âgés : 3 à 5 mois), en cases individuelles ;

- R5 = 15 sujets (les plus jeunes : 1 à 2 mois), en 3 sous-groupès de 4 et 1 sousgroupe de 3 , avec dans chacun d'entre eux une femelle du même âge.

L'effet d'un âge de réunion plus précoce que dans l'expérience 2, des animaux élevés en groupe constituait le principal objectif de cette troisième expérimentation. 
Dans ces deux dernières expériences, les animaux ont été logés dans le même bâtiment et ont reçu une alimentation identique constituée d'orge, de mélasse et de condiment minéral vitaminé, complétés après l'âge de 7 mois par de l'avoine, du foin et de la paille.

\section{B. Collectes de sperme}

Elles ont été effectuées à l'aide d'un vagin artificiel adapté à l'espèce, dans un parc extérieur à ceux d'élevage. Une femelle étrangère aux groupes, maintenue en contention et dont la réceptivité sexuelle était induite par une injection de Benzoate d'Oestradiol était utilisée comme boute en train.

Dans les groupes R1, R2 et R3, 8 essais de collecte de semence ont été réalisés en juillet, puis 15 en septembre-octobre.

Dans les groupes R4 et R5, 15 épreuves ont été réalisées en septembre-octobre.

Dans les groupes N1 et N2, 15 épreuves ont également été effectuées en décembrejanvier.

Selon CoRTEEL (1981), 15 éjaculats sont nécessaires pour une bonne estimation de la capacité de production spermatique de la vie sexuelle de l'animal.

Dans tous les cas, ces épreuves se sont déroulées au rythme de 2 par semaine et elles duraient 3 minutes au maximum, ou étaient interrompues après l'obtention d'un éjaculat.

\section{Critères de mesure}

Le pourcentage d'éjaculats obtenus par rapport au nombre d'épreuves a été pris en considération dans les trois expériences.

La latence à la première monte, à l'éjaculation et le nombre de montes avant éjaculation ont été pris en compte dans l'expérience 1.

Le volume de semence, la concentration en spermatozoïdes par millilitre et le nombre total de spermatozoïdes ont été déterminés, dans les 3 expériences.

Le pourcentage d'éjaculats éliminés pour un volume insuffisant $(<0,1 \mathrm{ml})$ ou une mauvaise concentration $\left(<1,9 \times 10^{9}\right.$ spermatozoïdes par $\left.\mathrm{ml}\right)$, d'une part, et la qualité de la semence estimée 5 et 120 minutes après dégel d'une paillette de semence à $37^{\circ} \mathrm{C}$, d'autre part, l'ont été dans les 2 dernières.

Le volume testiculaire a été estimé toutes les 2 semaines, par palper comparatif avec un chapelet de boules ovoïdes de volume connu, dans les expériences 1 et 3 .

\section{Analyse des résultats}

Les tests statistiques suivants ont été utilisés pour l'analyse des données :

- test $\mathbf{U}$ de Mann et Whitney pour les comparaisons des critères spermatiques et de nombre de montes (SIEGEL, 1956); 
- test de $\mathrm{Chi}^{2}$ pour les pourcentages d'éjaculats obtenus (SIEGEL, 1956); 1980).

- test $\mathrm{t}$ de Student pour les comparaisons de latences (Snedecor \& Cochran,

\section{Résultats}

\section{A. Comportement sexuel à la collecte}

Dans l'expérience 1, tous les mâles ont produit de la semence au moins une fois, mais leur efficacité était très différente selon le groupe d'élevage.

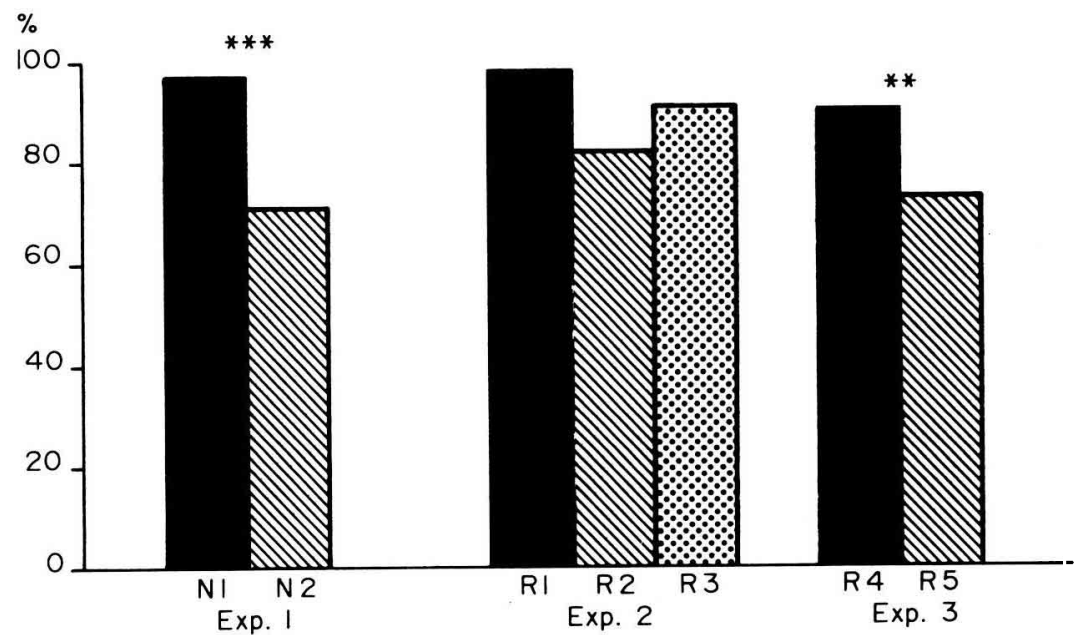

Fig. 1

Pourcentage d'éjaculations obtenues après 15 sollicitations, chez des jeunes boucs soumis à différentes conditions d'élevage.

Percentage of achieved ejaculations after 15 attempts, in young male goats under different rearing conditions.

N1 = mâles en groupes, réunis quelques jours après la naissance males grouped a few days after birth

$\mathrm{N} 2=$ mâles en groupes, réunis à l'âge de 8 à 11 mois

males grouped between 8 and 11 months of age

$\mathrm{R} 1=$ mâles en cases individuelles males reared in individual pens

R2 = mâles en groupes, sans femelle, réunis à l'âge de 2 à 4,5 mois males grouped, without female, between 2 and 4.5 months of age

R3 = mâles en groupes, avec femelles, réunis à l'âge de 2 à 4,5 mois males grouped, with females, between 2 and 4.5 months of age

R4 = mâles en cases individuelles males reared in individual pens

R5 = mâles en groupes, avec femelles, réunis à l'âge de 1 à 2 mois males grouped, with females, between 1 and 2 months of age

${ }^{* * *}=\mathrm{P}<0,001 ;{ }^{* *}=\mathrm{P}<0,01$. 


\section{Tableau 1}

Comportement sexuel de jeunes boucs soumis à différentes conditions d'élevage, lors d'essais de collectes de semence au vagin artificiel.

Sexual behaviour at semen collection of young male goats reared under different conditions.

\begin{tabular}{|c|c|c|c|c|c|c|c|}
\hline \multirow{2}{*}{$\begin{array}{l}\text { Conditions d'élevage } \\
\text { Rearing conditions }\end{array}$} & \multicolumn{2}{|c|}{$\begin{array}{l}\text { Expérience } 1 \\
\text { Experiment }\end{array}$} & \multicolumn{3}{|c|}{$\begin{array}{l}\text { Expérience } 2 \\
\text { Experiment }\end{array}$} & \multicolumn{2}{|c|}{$\begin{array}{l}\text { Expérience } 3 \\
\text { Experiment }\end{array}$} \\
\hline & $\mathrm{N} 1$ & $\mathrm{~N} 2$ & R1 & $\mathbf{R} 2$ & R3 & R4 & R5 \\
\hline $\begin{array}{l}\text { Nombre de mâles par groupe ... } \\
\text { Number of males per group }\end{array}$ & 8 & 10 & 9 & 9 & 9 & 14 & 15 \\
\hline $\begin{array}{l}\text { Latence (en sec.) à l'éjaculation . } \\
\text { Latency (sec.) at ejaculation }\end{array}$ & $\begin{array}{r}42,3 \\
\pm 6,9\end{array}$ & $\begin{array}{r}62,2 \\
\pm 8,8\end{array}$ & & & & & \\
\hline $\begin{array}{l}\text { Nombre de montes pour } \\
1 \text { éjaculat } \\
\text { Number of matings per } \\
\text { one ejaculate }\end{array}$ & $\begin{array}{r}1,8^{*} \\
\pm 0,2\end{array}$ & $\begin{array}{r}2,9^{*} \\
\pm 0,4\end{array}$ & & & & & \\
\hline $\begin{array}{l}\% \text { d'éjaculats obtenus pour } \\
15 \text { sollicitations } \\
\% \text { of ejaculates per } 15 \text { attempts }\end{array}$ & $97^{* * *}$ & $71^{* * *}$ & $97,8^{*}$ & $82,1^{*}$ & 91,4 & $90^{* *}$ & $73^{* *}$ \\
\hline \multicolumn{8}{|c|}{$\begin{aligned} \mathrm{N} 1= & \text { mâles en groupes, réunis quelques jours après la naissance. } \\
& \text { males grouped a few days after birth. } \\
\mathrm{N} 2= & \text { mâles en groupes, réunis à l'âge de } 8 \text { à } 11 \text { mois. } \\
& \text { males grouped between } 8 \text { and } 11 \text { months of age. }\end{aligned}$} \\
\hline \multicolumn{8}{|c|}{$\begin{aligned} \mathrm{R} 1= & \text { mâles en cases individuelles. } \\
& \text { males reared in individual pens. } \\
\mathrm{R} 2= & \text { mâles en groupes, sans femelle, réunis à l'âge de } 2 \text { à } 4,5 \text { mois. } \\
& \text { males grouped, without female, between } 2 \text { and } 4,5 \text { months of age. } \\
\mathrm{R} 3= & \text { mâles en groupes, avec femelles, réunis à l'âge de } 2 \text { à } 4,5 \text { mois. } \\
& \text { males grouped, with females, between } 2 \text { and } 4,5 \text { months of age. }\end{aligned}$} \\
\hline \multicolumn{8}{|c|}{$\begin{array}{l}\mathrm{R} 4=\text { mâles en cases individuelles. } \\
\text { males reared in individual pens. } \\
\mathrm{R} 5=\text { mâles en groupes, avec femelles, réunis à l'âge de } 1 \text { à } 2 \text { mois. } \\
\quad \text { males grouped, with females, between } 1 \text { and } 2 \text { months of age. } \\
* \mathrm{P}<0,05 ; * * \mathrm{P}<0,001 ;{ }^{* * *} \mathrm{P}<0,001 . \\
\pm: \text { écart-type à la moyenne; } \pm \text { : standard deviation. }\end{array}$} \\
\hline
\end{tabular}

Le taux d'éjaculations obtenues par rapport au nombre de sollicitations a été de $97 \%$ dans le groupe N1 (réunis tôt), contre $71 \%$ dans le groupe N2 (réunis après la puberté), où seulement 6 des 10 animaux ont pu être collectés régulièrement $(\mathrm{P}<0,001)$. De plus, les conditions de collecte ont parfois dû être modifiées pour certains sujets de ce groupe $\mathrm{N} 2$ (chèvre boute en train libre ou même introduite dans le parc d'élevage), alors qu'elles sont restées standardisées pour les mâles du groupe N1.

Par contre, la latence moyenne à la première monte, lorsqu'il y a eu éjaculation n'a pas différé entre les deux groupes $(\mathrm{N} 1=34,4 \mathrm{sec}$. ; $\mathrm{N} 2=33,3 \mathrm{sec}$. $)$.

Le nombre moyen de montes avant éjaculation dans le vagin artificiel a été significativement inférieur dans le groupe N1 (1,8 vs 2,$9 ; \mathrm{P}<0,05)$. Parallèlement, la latence à l'éjaculation a été inférieure dans ce groupe pour les 8 premières épreuves. Cependant, la différence n'est pas significative sur l'ensemble des 15 éjaculats $(\mathrm{N} 1=42,3$ sec. $; \mathrm{N} 2=62,2$ sec. $)$. 
Dans l'expérience 2, en tout début de saison sexuelle (juillet), le démarrage de l'activité sexuelle en situation de collecte de semence a été variable selon les groupes. Lors de la première épreuve, 3 boucs sur les 9 élevés individuellement (groupe R1) ont éjaculé dans le vagin artificiel, contre aucun sur les 18 élevés en groupes unisexués ou mixtes (R2 et R3).

Le taux de réussite en collecte de semence par rapport au nombre de sollicitations a été de $41,7 \%$ en $\mathrm{R} 1$ contre $10,6 \%$ en $\mathrm{R} 2$ et $\mathrm{R} 3$ réunis $(\mathrm{P}<0,001)$. Ces deux derniers groupes ne diffèrent pas entre eux.

Lors des 15 épreuves effectuées ultérieurement (septembre-octobre), tous les mâles ont été collectés. Certains l'ont cependant été irrégulièrement, puisque le taux de réussite a été de $97,8 \%$ en $\mathrm{R} 1$ contre $82,1 \%(\mathrm{P}<0,05)$ en $\mathrm{R} 2$ et $91,4 \%$ en $\mathrm{R} 3$ respectivement.

Dans l'expérience 3, $90 \%$ des essais de collecte de semence ont été couronnés de succès dans le groupe R4 (mâles en cases individuelles) contre $73 \%$ dans le R5 (mâles en groupes mixtes), au cours des 15 épreuves. La différence est significative $(P<0,01)$.

\section{B. Production spermatique}

Dans l'expérience 1, le volume moyen de semence par éjaculat est supérieur significativement dans le groupe d'animaux réunis tôt $(\mathrm{N} 1)(0,80 \mathrm{ml}$ vs $0,58 \mathrm{ml}$ pour ceux réunis après la puberté ; $\mathbf{P}<0,05)$.

La concentration moyenne en spermatozoïdes par $\mathrm{ml}$ de semence ne diffère pas de manière significative entre les 2 groupes $\left(\mathrm{N} 1=2,94 \times 10^{9} ; \mathrm{N} 2=3,10 \times 10^{9}\right)$. Toutefois, la différence entre le nombre moyen total de spermatozoïdes par éjaculat des deux groupes est proche du seuil de signification de $5 \%\left(\mathrm{~N} 1=2,23 \times 10^{9} ; \mathrm{N} 2=1,79 \times 10^{9}\right)$, en faveur du groupe réuni tôt.

Dans l'expérience 2, le volume moyen par éjaculat n'est pas significativement différent entre les mâles élevés individuellement (R1) et ceux des groupes unisexués (R2) ou mixtes (R3) $(0,89 \mathrm{ml}$ versus $1,02 \mathrm{ml}$ et $0,81 \mathrm{ml}$ respectivement). Par contre, il diffère entre ces deux derniers groupes $(P<0,05)$.

La concentration moyenne en spermatozoïdes par $\mathrm{ml}$ est significativement plus élevée pour les boucs du lot R1 $\left(3,68 \times 10^{9}\right)$ que pour ceux des 2 autres groupes $(P<0,05)$. Ces derniers ne diffèrent pas entre eux (respectivement 2,93 et $\left.3,29 \times 10^{9}\right)$.

Le pourcentage d'éjaculats éliminés à cause d'un volume ou/et d'une concentration insuffisants est significativement plus important pour les mâles du lot R2 que pour ceux de $\mathrm{R} 1(8,2 \%$ vs $0,8 \% ; \mathrm{P}<0,05)$. Ce pourcentage est intermédiaire pour les sujets de R3 $(4,1 \%)$ et ne diffère pas de manière significative des deux autres.

Aucune différence entre les trois groupes n'a été observée en ce qui concerne la quantité totale de spermatozoïdes émis et la qualité de la semence observée 5 et 120 minutes après dégel d'une paillette à $37^{\circ} \mathrm{C}$.

Dans l'expérience 3, volume moyen, concentration en spermatozoïdes par $\mathrm{ml}$ et nombre total de spermatozoïdes par éjaculat sont significativement inférieurs dans le 
TABleau 2

Production spermatique de jeunes boucs soumis à différentes conditions d'élevage. Semen production of young male goats reared under different conditions.

\begin{tabular}{|c|c|c|c|c|c|c|c|}
\hline \multirow{2}{*}{$\begin{array}{l}\text { Conditions d'élevage } \\
\text { Rearing conditions }\end{array}$} & \multicolumn{2}{|c|}{$\begin{array}{l}\text { Expérience } 1 \\
\text { Experiment }\end{array}$} & \multicolumn{3}{|c|}{$\begin{array}{c}\text { Expérience } 2 \\
\text { Experiment }\end{array}$} & \multicolumn{2}{|c|}{$\begin{array}{c}\text { Expérience } 3 \\
\text { Experiment }\end{array}$} \\
\hline & N1 & $\mathrm{N} 2$ & R1 & $\mathrm{R} 2$ & R3 & $\mathrm{R} 4$ & R5 \\
\hline $\begin{array}{l}\text { Nombre de mâles par groupe } . . \\
\text { Number of males per group }\end{array}$ & 8 & 10 & 9 & 9 & 9 & 14 & 15 \\
\hline $\begin{array}{l}\text { Volume de semence }(\mathrm{en} \mathrm{ml}) \ldots \\
\text { Semen volume }\end{array}$ & $\begin{aligned} & 0,80^{*} \\
\pm & 0,03\end{aligned}$ & $\begin{array}{r}0,58^{*} \\
\pm 0,03\end{array}$ & $\begin{array}{r}0,89 \\
\pm 0,18\end{array}$ & $\begin{aligned} & 1,02^{*} \\
\pm & 0,27\end{aligned}$ & $\begin{array}{r}0,81^{*} \\
\pm 0,17\end{array}$ & $\begin{array}{r}0,86^{*} \\
\pm 0,43\end{array}$ & $\begin{aligned} & 0,74^{*} \\
\pm & 0,29\end{aligned}$ \\
\hline $\begin{array}{l}\text { Concentration en } \mathrm{spz} / \mathrm{ml} \times 10^{9} \\
\text { Concentration }(\mathrm{spz} / \mathrm{ml})\end{array}$ & $\begin{array}{r}2,94 \\
\pm 0,10\end{array}$ & $\begin{array}{r}3,10 \\
\pm 0,10 \\
h^{*}\end{array}$ & $\begin{array}{r}3,68^{*} \\
\pm 0,50\end{array}$ & $\begin{array}{r}2,93^{*} \\
\pm 0,81\end{array}$ & $\begin{array}{r}3,29^{*} \\
\pm 0,61\end{array}$ & $\begin{array}{r}3,42^{*} \\
\pm 0,79\end{array}$ & $\begin{aligned} & 2,90^{*} \\
\pm & 1,0\end{aligned}$ \\
\hline $\begin{array}{l}\text { Nombre total de } \mathrm{spz} \times 10^{9} \\
\text { Total number of } s p z\end{array}$ & $\begin{array}{r}2,23 \\
\pm 0,10\end{array}$ & $\begin{array}{r}1,79 \\
\pm 0,14\end{array}$ & $\begin{array}{ll} & 3,27 \\
\pm & 1,5\end{array}$ & $\begin{aligned} & 2,98 \\
\pm & 1,4\end{aligned}$ & $\begin{aligned} & 2,66 \\
\pm & 1,4\end{aligned}$ & $\begin{aligned} & 2,84^{*} \\
\pm & 1,4\end{aligned}$ & $\begin{aligned} & 2,24^{*} \\
\pm & 1,3\end{aligned}$ \\
\hline $\begin{array}{l}\% \text { d'éjaculats éliminés } \ldots . . . . \\
\% \text { of eliminated ejaculats }\end{array}$ & & & $0,8^{*}$ & $8,2^{*}$ & 4,1 & 4 & 7 \\
\hline $\begin{array}{l}\text { Qualité de la semence } 5 \text { et } 120 \mathrm{mn} \\
\text { après dégel . } \ldots \ldots \text { a } 120 \text { min after } \\
\text { Semen quality } 5 \text { and } \\
\text { thawing }\end{array}$ & \multicolumn{7}{|c|}{$\begin{array}{l}\text { aucune différence } \\
\text { no difference }\end{array}$} \\
\hline \multicolumn{8}{|c|}{$\begin{aligned} \mathrm{N} 1= & \text { mâles en groupes, réunis quelques jours après la naissance. } \\
& \text { males grouped a few days after birth. } \\
\mathrm{N} 2= & \text { mâles en groupes, réunis à l'âge de } 8 \text { à } 11 \text { mois. } \\
& \text { males grouped between } 8 \text { and } 11 \text { months of age. }\end{aligned}$} \\
\hline \multicolumn{8}{|c|}{$\begin{aligned} \mathrm{R} 1= & \text { mâles en cases individuelles. } \\
& \text { males in individual pens. } \\
\mathrm{R} 2= & \text { mâles en groupes, sans femelle, réunis à l'âge de } 2 \text { à } 4,5 \text { mois. } \\
& \text { males grouped, without female, between } 2 \text { and } 4,5 \text { months of age. } \\
\mathrm{R} 3= & \text { mâles en groupes, avec femelles, réunis à l'âge de } 2 \text { à } 4,5 \text { mois. } \\
& \text { males grouped, with females, between } 2 \text { and } 4,5 \text { months of age. }\end{aligned}$} \\
\hline \multicolumn{8}{|c|}{$\begin{aligned} \mathrm{R} 4= & \text { mâles en cases individuelles. } \\
& \text { males in individual pens. } \\
\mathrm{R} 5= & \text { mâles en groupes, avec femelles, réunis à l'âge de } 1 \text { à } 2 \text { mois. } \\
& \text { males grouped, with females, between } 1 \text { and } 2 \text { months of age. }\end{aligned}$} \\
\hline
\end{tabular}

groupe $\mathrm{R5}$ (respectivement : 0,74 vs 0,$86 ; \mathrm{P}<0,05 ; 2,90$ vs 3,$42 ; \mathrm{P}<0,05 ; 2,24$ vs $2,84 ; \mathrm{P}<0,05)$.

Par contre, aucune différence n'a été notée pour un pourcentage d'éjaculats éliminés à cause d'un volume et/ou une concentration trop faibles, et pour la qualité de la semence 5 et 120 minutes après dégel d'une paillette à $37^{\circ} \mathrm{C}$.

\section{Volume testiculaire}

Dans l'expérience 1 , le volume moyen des testicules des mâles réunis après la puberté, est supérieur pendant toute l'expérience, mais la différence se réduit à la fin 
(début : $\mathrm{P}<0,001$; fin : $\mathbf{P}<0,05$ ). La décroissance, normale à cette époque est moins prononcée pour les animaux réunis à la naissance.

Dans l'expérience 3, les jeunes boucs en cases individuelles, ont un volume testiculaire moyen supérieur à celui des mâles en groupes, pendant la période de sollicitation à la collecte de semence $(\mathrm{P}<0,01)$. La différence d'âge entre les deux groupes expérimentaux peut en être la cause principale.

\section{Discussion}

Les expériences 2 et 3 confirment que l'isolement en cases individuelles, lors de la période qui précède la puberté, n'affecte pas la capacité de réaction sexuelle dans les conditions de collecte de sperme au vagin artificiel. Au contraire, les mâles placés dans ces conditions ont été les plus précoces et les plus réguliers dans leur comportement en collecte.

Ces résultats sont en accord avec les observations de BRYANT (1975) chez le bélier, et de LANE et al. (1983) chez le taureau. Ils sont opposés à ceux rapportés par Hemsworth et al. $(1981,1983)$ chez le verrat, et Sylver \& Price (1986), chez le taureau, où l'influence négative de l'isolement est toutefois partielle et de durée limitée.

La production spermatique, notamment la quantité de spermatozoïdes éjaculés, ne paraît pas très affectée par les conditions d'élevage. Dans l'expérience 3 , elle est supérieure pour les animaux entretenus en cases individuelles, mais ils sont plus âgés que ceux élevés en groupes. Dans l'expérience 2, seul le pourcentage d'éjaculats éliminés pour un volume et/ou une concentration trop faible, est significativement inférieure pour les mâles élevés seuls. Ces résultats sont proches de ceux obtenus précédemment chez le bouc, où la production spermatique d'animaux entretenus en cases individuelles ou en groupes mixtes ne différait pas notablement (OrGEUR et al., 1984 b).

L'expérience 2 montre, par ailleurs, que dans l'élevage en groupe, la présence de femelles n'a pas un effet déterminant.

Sur le plan comportemental, les mâles entretenus en mixité ont toutefois, dans nos conditions, un taux d'éjaculation plus élevé que ceux privés de contact avec des femelles mais la différence n'est pas significative. Chez les Ovins, Pretorius (1967), Winfield \& Makin (1978), Orgeur et al. (1984 a), Casteilla et al. (1987), rapportent une influence très favorable d'un mode d'élevage hétérosexué dans la période prépubère, sur le démarrage de l'activité copulatoire des jeunes mâles.

Sur le plan spermatique, si la quantité moyenne de spermatozoïdes émis n'est pas influencée par les conditions d'élevage, le nombre d'éjaculats éliminés pour un volume insuffisant ou une mauvaise concentration est moins important s'il y a eu présence plutôt qu'absence de femelles.

L'hypothèse d'une stimulation de la sécrétion de testostérone par la présence de femelles rapportée par Illius et al. (1976), Schanbacher et al. (1987) et Gonzalez et al. (sous presse), chez le bélier, ne peut être retenue ici pour expliquer une action sur le développement testiculaire et ses conséquences sur la production spermatique, dans la mesure où les différences, peu marquées, sont en faveur de l'isolement. 
Les résultats de la première expérience montrent que la constitution précoce du groupe social a un effet favorable sur les performances sexuelles et la production spermatique des jeunes boucs, bien que ceux-ci aient un volume testiculaire inférieur, en comparaison d'une réunion postérieure à la puberté. A notre connaissance, aucune étude n'a été faite sur ce sujet dans l'espèce caprine. Par contre, il a été établi chez des bovins femelles (BouIssou \& Hövels, 1976) que la précocité de la constitution du groupe social induisait une grande tolérance interindividuelle. Ce phénomène pourrait réduire l'effet défavorable résultant chez certains sujets de leur statut de subordonnés, comme Lindsay et al. (1976) et Jennings (1977) l'ont observé chez les Ovins.

Au vu des résultats de l'expérience 3 , il apparaît que la réunion des sujets entre 1 et 2 mois d'âge n'apporte pas d'amélioration, comparativement à des animaux groupés plus tardivement (à l'âge de 2 à 4,5 mois : expérience 2). Toutefois, lors de la constitution des deux groupes expérimentaux de cette troisième étude, les mâles en cases individuelles étaient, pour des raisons pratiques, les plus âgés. Nous ne pouvons exclure un effet de ce facteur sur la production spermatique, voire même sur le comportement en collecte, dans la mesure où la taille testiculaire était inférieure pour les animaux en groupes, lors des sollicitations à la collecte. Les réserves épididymaires devaient être, de ce fait, moins importantes.

Les difficultés de mise en auvre de l'activité sexuelle en collecte de semence au vagin artificiel, pour les sujets élevés en groupes, pourraient résulter en partie, des conditions mêmes des épreuves, différentes selon le mode d'élevage :

- d'une part, l'intervention directe de l'homme pour sortir et réintégrer l'animal dans son parc d'élevage, lors des manipulations afférentes à ces épreuves de sollicitation à la collecte est très limitée pour les mâles entretenus seuls : l'ouverture de la porte de leur case leur permet de se déplacer sans contrainte vers le lieu de collecte ;

- d'autre part, la perturbation résultant, au moment de l'épreuve, de la séparation d'avec les congénères peut avoir un effet anxiogène par rapport aux animaux qui vivent habituellement dans l'isolement.

Une procédure différente lors des expérimentations futures devrait permettre de vérifier cette hypothèse.

En conclusion, d'un point de vue pratique, nous avons confirmé que l'élevage en cases individuelles permet la meilleure expression des capacités sexuelles des jeunes boucs.

Cependant, nos résultats montrent également que l'élevage en groupes ne doit pas être pour autant abandonné : il n’a pas de conséquence défavorable majeure, si certaines précautions sont prises. Un élément essentiel paraît être l'âge auquel est constitué le groupe, événement qui doit avoir lieu le plus précocement possible.

Par ailleurs, la présence de femelles est préférable à leur absence.

Les conditions de manipulation, et notamment d'habituation à la présence de l'homme devront être précisées.

En résumé, les résultats présentés ici, nous laissent espérer qu'une méthode d'élevage en groupes hétérosexués sera possible chez les Caprins, en tenant compte à la fois des conditions déjà évoquées et de celles qui restent à préciser. Cela permettrait une réduction substantielle du coût d'entretien des mâles de cette espèce.

Reçu en avril 1988.

Accepté en juillet 1988. 


\section{Remerciements}

Nous exprimons notre gratitude à MM. VENIER et PIGNON dont l'assistance technique a été précieuse lors de l'expérience 1, ainsi qu'à tout le personnel de la Station Expérimentale d'Insémination Artificielle de Rouillé, notamment MM. Bernolas et Berson, participants actifs aux expériences 2 et 3 .

\section{Summary \\ Effect of social experience during development upon sexual behaviour and semen production in young male goats}

Young male goats selected for semen production in artificial insemination are usually reared in individual pens, as their behaviour during semen collection as well as their sperm production are reported to be impaired when kept in unisexual groups.

However, such a rearing method is expensive as it requires special adapted housing and important human labour.

Three experiments (involving a total of 74 subjects) were designed to test the effect of rearing the young males in hetero- or unisexual groups, and the effect of age at grouping compared with rearing in individual pens.

The results support the observation that rearing in individual pens results in an improved sexual capacity. Sperm production, especially the semen quality is improved (Tables 1 and 2).

Moreover, results show that social grouping at birth has a favourable effect both upon sexual behaviour at semen collection and semen production when compared with postpubertal grouping. When young males were housed together from birth (Group 1, experience 1), an ejaculation was obtained in $97 \%$ of the semen collection attempts, with a greater number of spermatozoa, compared with only $71 \%$ when grouping took place after the age of 10 months (group 2, Figure 1, Table 2).

On the other hand, grouping the subjects between one and two months of age did not appear as better than later ( $2-4.5$ months).

The presence of females in the group had a favourable effect.

It may be concluded that rearing in heterosexual group is possible without markedly impairing the sexual performance, especially when grouping takes place as early as possible. Such results are of economic importance, as they could allow a reduction of the costs of maintenance of young male goats.

However, husbandry conditions, especially man-animal relationships and manipulations are of importance and require further studies.

Key words : Male Goats, development, social experience, male sexual behaviour, semen production.

\section{Références bibliographiques}

Bouissou M.F., Hövels J., 1976. Effet d'un contact précoce sur quelques aspects du comportement social des bovins domestiques. Biol. Behav., 1, 17-36.

Bryant M.J., 1975. A note on the effect of rearing experience upon the development of sexual behaviour in ram lamb. Anim. Prod., 21, 97-99. 
Casteilla L., Orgeur P., Signoret J.P., 1987. Effects of rearing conditions on sexual performance in the ram : practical use. Appl. Anim. Behav. Sci., 19, 111-118.

CorTeEL J.M., 1981. Collection, processing and artificial insemination of goats semen. In : "Goat production » G. Gall Ed., Acad. Press, 171-191.

Gonzalez R., Poindron P., Signoret J.P. Temporal variation in LH and testosterone responses of rams after the introduction of oestrous females during the breeding season. J. Reprod. Fert., 83 (sous presse).

Hemsworth P.H., Winfield C.G., Chamley W.A., 1981. The influence of the presence of the female on the sexual behaviour and plasma testosterone levels on the mature male pig. Anim. Prod., 32, 61-65.

Hemsworth P.H., Winfield C.G., Hansen C., Makin A.W., 1983. The influence of isolation from females and mating frequency on the sexual behaviour and semen quality of young postpubertal boars. Anim. Prod., 37, 49-52.

Illius A.W., Haynes N.B., Purvis K., Lamming G.E., 1976. Plasma concentration of testosterone in the developing ram in different social environments. J. Reprod. Fertil., 48, 17-24.

JeNnings J.J., 1977. Influence of mating behaviour of rams on fertility in progestagen-PMSTreated anestrous ewes. Irish J. Agric. Res., 16, 155-162.

Lane S.M., Kiracofe G.H., Craig J.V., Schalles R.R., 1983. The effects of rearing environment on sexual behavior of young beef bulls. J. Anim. Sci., 57, 1084-1089.

LE Roux P.J., Barnard J.P., 1974. The effect of heterosexual contact on libido and mating dexterity in Karakul rams. S. Afr. J. Anim. Sci., 4, 171-174.

Lindsay D.R., Dunsmore D.G., Williams J.D., Syme G.J., 1976. Audience effects on the mating behaviour of rams. Anim. Behav., 24, 818-821.

Montigny (de) G., Lequenne D., 1975. Observations sur la croissance et le comportement sexuel de jeunes boucs élevés en lots. $I^{\text {res }}$ Journées de la Recherche Ovine et Caprine. INRAITOVIC., 18-22.

Orgeur P., Signoret J.P., 1984. Sexual play and its functional significance in the domestic sheep (Ovis aries L.). Physiol. Behav., 33, 111-118.

Orgeur P., Venier G., Signoret J.P., 1984 a. Effets de l'environnement social au cours du développement sur l'apparition et l'intensité de l'activité sexuelle du jeune bélier. Ann. Zootech., 33, 1-18.

Orgeur P., Signoret J.P., Leboeuf B., $1984 \mathrm{~b}$. Effects of rearing conditions upon the development of sexual activity and semen production in young male goats. In : "The male in farm animal reproduction ». Courot M., Ed., Symp. C.E.C., 135-140.

Pretorius P.S., 1967. Libido and mating dexterity in rams reared and kept in isolation from ewes. Proc. S. Afr. Soc. Anim. Prod., 208-212.

Price E.O., Sмrтн V.M., 1984. The relationship of male-male mounting to mate choice and sexual performance in male dairy goats. Appl. Anim. Behav. Sci., 13, 71-82.

Schanbacher B.D., Orgeur P., Pelletier J., Signoret J.P., 1987. Behavioural and hormonal responses of sexually-experienced Ile-de-France rams to oestrous females. Anim. Reprod. Sci., 14, 293-300.

SiEgEL S., 1956. Non parametric statistics for the behavioral sciences. McGraw Hill, New York.

Snedecor G.W., Cochran W.G., 1980. Statistical Methods. 7th Ed. The Iowa State University Press.

Sylver G.V., Price E.O., 1986. Effects of individual vs group rearing on the sexual behavior of prepuberal beef bulls : mount orientation and sexual responsiveness. Appl. Anim. Behav. Sci., 15, 287-294.

Winfield C.G., Makin A.W., 1978. A note on the effect of continuous contact with ewes showing regular oestrus and of post-weaning growth rate on the sexual activity of Corriedale rams. Anim. Prod., 27, 361-364.

Zenchak J.J., ANDERson G.C., Schein M.W., 1981. Sexual partner preference of adult rams as affected by social experiences during rearing. Appl. Anim. Ethol., 7, 157-167. 